\title{
Magnetooptical and Reflectivity Studies of $\mathrm{SrTiO}_{3}$
}

\author{
Katsuaki Sato and Takayuki Ishibashi \\ Department of Applied Physics, Tokyo University of Agriculture and Technology, \\ Koganei, Tokyo 184-8588, Japan
}

Faraday rotation spectra were measured in single crystals of $\mathrm{SrTiO}_{3}$ between 1.7 and $3.2 \mathrm{eV}$ at room temperature and at $20 \mathrm{~K}$. The rotation showed a monotonous increase as the absorption edge was approached. In a single crystal with thickness of $0.5 \mathrm{~mm}$, it took a value as large as $7.7^{\circ}$ at $400 \mathrm{~nm}$ for magnetic field of $1 \mathrm{~T}$. The center of dispersion of the magneto-optical spectrum was determined to be $4.19 \mathrm{eV}$. This value agreed with the peak energy position $(=4.2 \mathrm{eV})$ of the imaginary part of dielectric function, $\varepsilon_{2}$ deduced from the reflectivity spectrum measured between 0.5 and $20 \mathrm{eV}$.

Key words: strontium titanate, Faraday rotation spectrum, reflectivity spectrum, isolator for short wavelength

\section{Introduction}

Recently short wavelength (blue to violet) laser diodes are appearing into the market. However, optical isolator materials suited for this wavelength region are not easily available. Magnetic garnets, most prevailing optical-isolator materials, are not transparent in the visible wavelengths due to strong absorption band caused by charge-transfer transitions. Although enhanced magnetooptical effect is observed in bismuth-substituted garnets, the figure of merit $F$ is not sufficient to be applied to optical isolators for wavelengths shorter than $1000 \mathrm{~nm}$, except for the transmission window around $800 \mathrm{~nm}$. For example a $\mathrm{Y}_{2.7} \mathrm{Bi}_{0.3} \mathrm{Fe}_{5} \mathrm{O}_{12}$ film shows a specific Faraday rotation of $9 \times 10^{4} \mathrm{deg} / \mathrm{cm}$ and an absorption coefficient of 5 $\times 10^{4} \mathrm{~cm}^{-1}$ at $400 \mathrm{~nm}$, which leads to $F$ of approximately 0.5 $\mathrm{deg} / \mathrm{dB}$. Since the Faraday rotation is known to be proportional to $\mathrm{Bi}$-content, one can get larger rotation by increasing $\mathrm{Bi}$ content. It was reported that Faraday rotation as large as $10^{6} \mathrm{deg} / \mathrm{cm}$ was obtained in $100 \% \mathrm{Bi}$-substituted garnet film, i.e., $\mathrm{Bi}_{3} \mathrm{Fe}_{5} \mathrm{O}_{12}(\mathrm{BIG})$ grown by ion beam sputtering on a $\mathrm{Gd}_{3} \mathrm{Ga}_{5} \mathrm{O}_{12}$ (GGG) substrate. ${ }^{1)}$ However even with this film $F$ is no more than $5 \mathrm{deg} / \mathrm{dB}$. This means that to get $45 \mathrm{deg}$ rotation for optical isolators, the insertion loss amounts to as large as $9 \mathrm{~dB}$. In addition, optical isolator requires thickness of $4.5 \times 10^{-5} \mathrm{~cm}=450 \mathrm{~nm}$, which exceeds critical thickness for pseudomorphic growth of the BIG film on GGG.

It is for this reason that optical isolators using diluted magnetic semiconductors (DMS) such as $\mathrm{Cd}_{1-\mathrm{x}} \mathrm{Mn}_{\mathrm{x}}$ Te and $\left(\mathrm{Hg}_{1-\mathrm{y}} \mathrm{Cd}_{\mathrm{y}}\right)_{1-\mathrm{x}} \mathrm{Mn}_{\mathrm{x}} \mathrm{Te}_{2}$ have been investigated for wavelengths in which magnetic garnets are not available, in spite of the demerit that they are in the paramagnetic phase or the spin-glass phase showing no spontaneous magnetization and require stronger magnetic field than garnets to be applied to isolators. The Verdet constant in commercially available $\mathrm{CdHgMnTe}$ takes a value of $\sim 4 \times 10^{-2} \mathrm{deg} / \mathrm{cmG}\left(\sim 2 \times 10^{2} \mathrm{deg} / \mathrm{cm}\right.$ for an applied field of
$5000 \mathrm{G}$ ) at $500 \mathrm{~nm}$. Although the band gap of the DMS can be swept from the IR to UV $(350 \mathrm{~nm})$ by varying $\mathrm{Mn}$ content, the wavelength region for practical application is limited to $\lambda>500 \mathrm{~nm}$ because of the absorption due to the $\mathrm{d}-\mathrm{d}$ transitions inherent to $\mathrm{Mn}^{2+}$ ion.

Only a few magnetic materials have been reported to show Faraday effect in short wavelength region at room temperature. $\mathrm{FeBO}_{3}$ and $\mathrm{FeF}_{3}$ were intensively studied materials since they are transparent in visible wavelengths and show considerably large Faraday rotation in short wavelengths. ${ }^{3)}$ Both $\mathrm{FeBO}_{3}$ and $\mathrm{FeF}_{3}$ are canted antiferromagnets with $T_{\mathrm{N}}=348 \mathrm{~K}$ and $365 \mathrm{~K}$, respectively. Absorption spectrum in a single crystal of $\mathrm{FeBO}_{3}$ locates the fundamental absorption edges at about $500 \mathrm{~nm}$ and two below-gap absorption peaks around $600 \mathrm{~nm}$ and $900 \mathrm{~nm}$. Faraday rotation shows a monotonous increase towards shorter wavelengths. At $525 \mathrm{~nm}$ where the absorption takes a minimum, the rotation is $2300 \mathrm{deg} / \mathrm{cm}$, corresponding to $\mathrm{F}=15 \mathrm{deg} / \mathrm{dB}$. On the other hand in $\mathrm{FeF}_{3}$ an absorption edge at $240 \mathrm{~nm}$ and $F=16 \mathrm{deg} / \mathrm{dB}$ at $400 \mathrm{~nm}$. However both materials suffer an optical birefringence as large as $10^{5}$ $\mathrm{deg} / \mathrm{cm}$, which makes practical applications of these materials quite difficult. In addition, they are not commercially available as single crystals of good quality.

We investigated magnetooptical effect in nonmagnetic materials. In 1967 Baer reported considerably large Faraday rotations in some of the perovskite transition metal oxides such as $\mathrm{SrTiO}_{3}, \mathrm{BaTiO}_{3}, \mathrm{KTaO}_{3}$ and $\mathrm{KTa}_{0.35} \mathrm{Nb}_{0.65} \mathrm{O}_{3}(\mathrm{KTN})$ as well as in rutile $\mathrm{TiO}_{2}{ }^{4)}$ Band gap energies are $3.40 \mathrm{eV}(365 \mathrm{~nm})$ in $\mathrm{SrTiO}_{3}$ at $296 \mathrm{~K}, 3.25 \mathrm{eV}$ $(381 \mathrm{~nm})$ in $\mathrm{BaTiO}_{3}$ at $403 \mathrm{~K}, 3.77 \mathrm{eV}(329 \mathrm{~nm})$ in $\mathrm{KTaO}_{3}$ at $296 \mathrm{~K}$ and $3.54 \mathrm{eV}(350 \mathrm{~nm})$ in $\mathrm{KTN}$ at $296 \mathrm{~K}$. He plotted spectra of $n \theta / H$ (Verdet constant multiplied by refractive constant) in these materials. The $n \theta / H$ of $\mathrm{SrTiO}_{3}$ at $3 \mathrm{eV}$ can be read from the plot as $-2.6 \times 10^{-2} \mathrm{deg} / \mathrm{cmG}$ for temperatures $296 \mathrm{~K}$ and $77 \mathrm{~K}$. Assuming $n=2.6$ Verdet constant can be obtained as $-1.0 \times 10^{-2} \mathrm{deg} / \mathrm{cmG}$. Other perovskites and rutile showed the similar magnitude of Faraday rotation. This study did not attract attention at that time, since single crystals of such oxides were not easily available at that time and there were no needs for short wavelength optical isolators. In 1970, Feldman et al. showed Faraday rotation in Bismuth oxide compounds, such as $\mathrm{Bi}_{12} \mathrm{GeO}_{20}, \mathrm{Bi}_{12} \mathrm{SiO}_{20}, \mathrm{Bi}_{12} \mathrm{TiO}_{20}$, etc. ${ }^{5)}$ According to their report Verdet constants of these compounds are nearly equal to $-2 \times 10^{-2} \mathrm{deg} / \mathrm{cmG}$ at $410 \mathrm{~nm}$. However these bismuth compounds show optical activity and cannot be selected as isolator materials.

Recently single crystals of $\mathrm{SrTiO}_{3}$ with a large size and a good crystalline quality become easily available as substrate materials for high-Tc superconductors. This 
prompted us to reinvestigate optical and magnetooptical properties of $\mathrm{SrTiO}_{3}$. In the present study we measured Faraday rotation spectra in good quality single crystals at room temperature as well as at $20 \mathrm{~K}$ and confirmed that the material show considerably large temperature-independent Faraday rotation in the short wavelength region around 400 $\mathrm{nm}$. To study the electronic origin of the magnetooptical effect we also measured the reflectivity spectrum for photon energies between 0.5 and $30 \mathrm{eV}$ with the help of synchrotron radiation and deduced dielectric functions using Kramers-Kronig relation.

\section{Experiments}

Specimens for the present study were single crystals of $\mathrm{SrTiO}_{3}$ obtained from different suppliers as substrates for epitaxial growth of high-Tc oxide superconductors. All of the crystals were cut parallel to the $\{100\}$ plane and mechanically polished. Sample thickness was typically 0.5 $\mathrm{mm}$. The samples were set on the cold finger of a helium refrigerator cryostat and were inserted between the pole pieces of an electromagnet, the maximum field of which is $1.2 \mathrm{~T}$. Faraday rotation spectra were measured using polarization modulation technique employing a photoeleastic modulator (PEM). A $150 \mathrm{~W}$ Xe lamp was used as a light source. The light was passed through a Glan Thomson polarizer whose transmission angle was set to make an angle of $45^{\circ}$ with the vertical axis and was focused by a lens through the PEM (Hinds inc. PEM90; modulation frequency was $50 \mathrm{kHz}$ ) on the sample surface. The light was then passed through an analyzer (Glan Thomson prism) and focused on the slit of a JASCO CT-25C monochromator with a 1200 lines $/ \mathrm{mm}$ grating blazed at $750 \mathrm{~nm}$. The light through the output slit was detected by a photomultiplier (Hamamatsu R928). The dc component of the output was kept constant by a feed-back circuit to a high voltage supply for the photomultiplier. The ac component was amplified using a lock-in amplifier tuned to the doubled frequency of the modulator. This method is in principle the same as described for a measurement of Kerr effect proposed by one of the authors (K.S.). ${ }^{6)}$ The calibration was carried out point by point by adjusting the angle of the analyzer to get the null signal.

Reflectivity spectra were measured using a Hitachi U-3410 spectrophotometer for energy region between 0.5 and $4 \mathrm{eV}$ and a Seya-Namioka monochromator combined with the synchrotron radiation (SR ring at Institute of Solid State Physics, University of Tokyo) for energies from 2 to $30 \mathrm{eV}$. Kramers-Kronig analysis was performed with the help of the optical constants determined for 2 to $5 \mathrm{eV}$ by a spectroscopic ellipsometer.

\section{Results and Discussion}

\subsection{Faraday rotation and optical transmission spectra}

$\mathrm{SrTiO}_{3}$ crystals show a negative Faraday rotation, which is proportional to the applied magnetic field. (Here, a positive sign is defined to be in the same direction as the current in the coils producing the magnetic field.) As shown by dots in Fig. 1 the Faraday rotation of a single crystal of

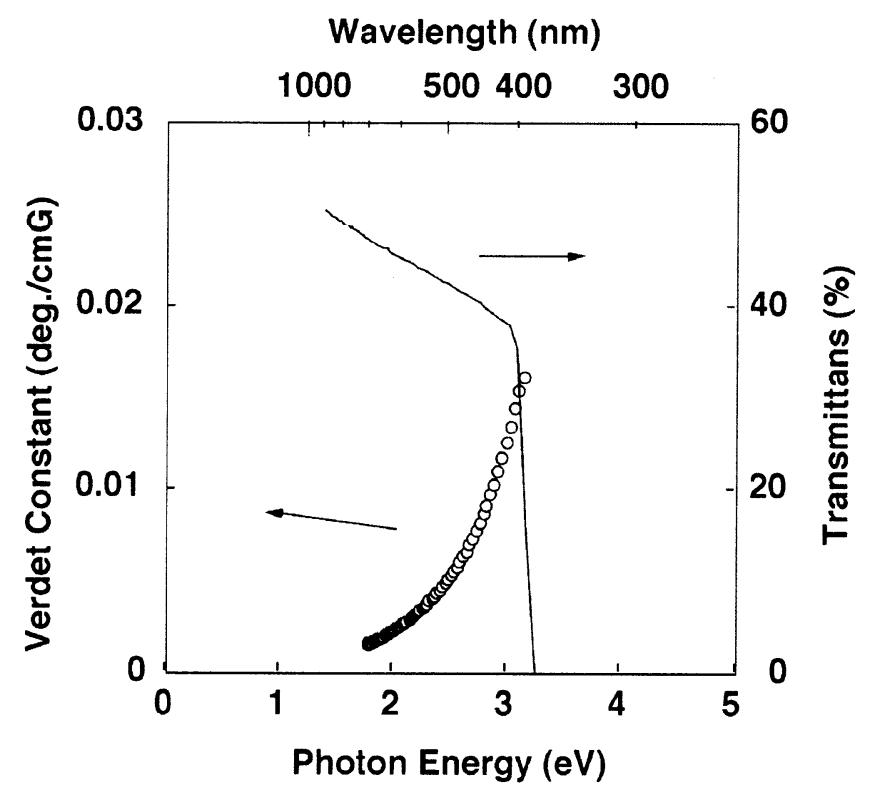

Fig. 1 Spectra of Faraday rotation and transmission of a $\mathrm{SrTiO}_{3}$ single crystal.

$\mathrm{SrTiO}_{3}$ increases monotonously with decrease of wavelength. The Verdet constant was determined as $-1.54 \times 10^{-2} \mathrm{deg} / \mathrm{cmG}$ at the wavelength of $400 \mathrm{~nm}$. The Faraday rotation should be compared with that of commercially available $\mathrm{CdHgMnTe}$ optical isolator materials, which was reported to be $+3 \sim 4 \times 10^{-2} \mathrm{deg} / \mathrm{cmG}$ at $980 \mathrm{~nm}$.

In order to investigate whether the magnetooptical effect is intrinsic or due to impurities or defects we measured in Fe-doped crystals and annealed crystals. No change in the value of the Faraday rotation was observed, from which we concluded that the effect is intrinsic.

The optical transmission spectrum of the $\mathrm{SrTiO}_{3}$ crystal $(0.5 \mathrm{~mm}$ in thickness) at room temperature is also shown in Fig. 1 by a solid curve. The Verdet constant shows a monotonous increase with photon energy up to 3.2 $\mathrm{eV}(-388 \mathrm{~nm})$, at which the material loses transparency. Optical transmission was found to be as small as $40 \%$ at $400 \mathrm{~nm}$. Such reduction of transmission may be attributed to surface reflection since refractive index of this material is fairly large. If $n=2.6$ is assumed the normal incidence reflectivity $R$ amounts to $19.7 \%$, thus leading to $T=(1-R)^{2}=0.64$ even if the material is assumed to be completely transparent. Such reflection may be minimized by suitable selection of antireflection coating. However, absorption coefficient after correction of reflection was estimated to be as large as $\sim 10 \mathrm{~cm}^{-1}$ at $400 \mathrm{~nm}$, corresponding to an insertion loss of $40 \mathrm{~dB} / \mathrm{cm}$. Such a large value of residual absorption may be attributed to intrinsic defects introduced during growth and surface damages due to polishing process and can be substantially reduced.

Using the Verdet constant of $1.54 \times 10^{-2} \mathrm{deg} / \mathrm{cmG}$ and the insertion loss of $40 \mathrm{~dB} / \mathrm{cm}$ we get figure of merit of $\mathrm{F}=3.85 \mathrm{deg} / \mathrm{dB}$ for applied field of $1 \mathrm{~T}$. Reduction of residual absorption is necessary. 


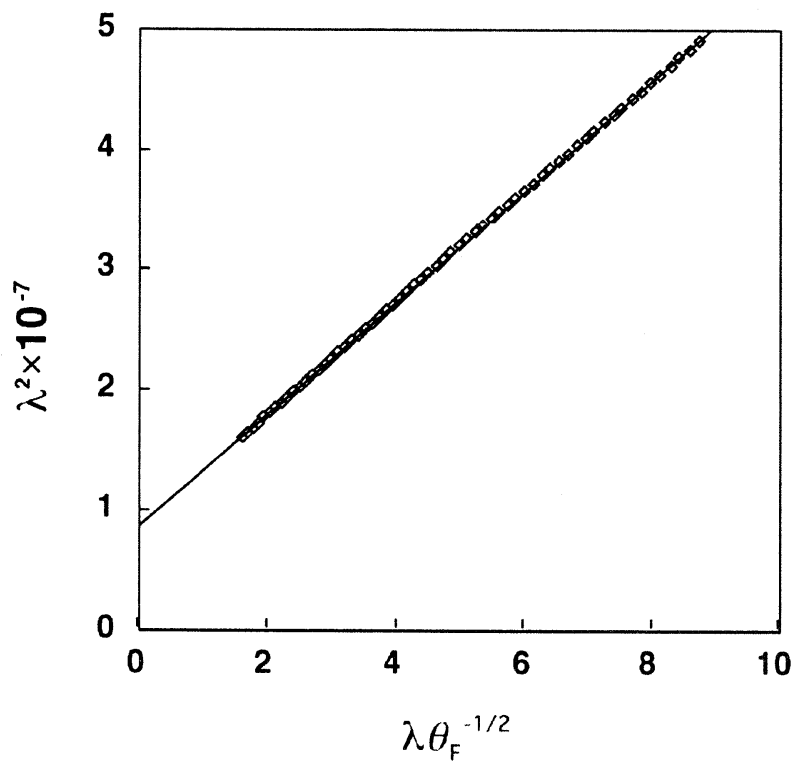

Fig. 2 Plot of $\lambda\left(\theta_{\mathrm{F}}\right)^{-1 / 2}$ vs. $\lambda^{2}$ in $\mathrm{SrTiO}_{3}$ for determination of parameters in eq (1).

\subsection{Determination of center of dispersion of the Faraday effect}

In order to determine the energy of optical transition from which Faraday rotation originates, we used the following dispersion relation ${ }^{7)}$ for the spectral dependence of Faraday rotation;

$$
\theta_{l}=A / \lambda^{2}\left(1-\lambda_{0}^{2} / \lambda^{2}\right)^{2}
$$

where $\lambda$ is wavelength, $\lambda_{0}$ center wavelength of dispersion and $A$ constant. The value of $\lambda_{0}$ can be determined by $\lambda\left(\theta_{\mathrm{F}}\right)^{-1 / 2}$ vs. $\lambda^{2}$ plot shown in Fig. 2, from which the photon energy for the center of dispersion was determined as 4.19 $\mathrm{eV}$. This value is larger than that of Baer's analysis in which the center of dispersion was determined as $3.4 \mathrm{eV}$ by using a sophisticated formula for Faraday effect of direct band gap materials.

\subsection{Temperature dependence}

The Faraday rotation spectra measured at $20 \mathrm{~K}$ and room temperature are shown in Fig. 3, from which it is found that temperature dependence of the rotation is negligible. This result is also consistent with the Baer's report. The temperature-independent Faraday rotation suggests that this effect does not originate from any paramagnetic centers, for which strong temperature dependence and positive sign are expected. The small temperature dependence observed above is quite favorable for optical isolator application.

\subsection{Dielectric functions deduced from reflectivity spectrum}

The negative rotation in $\mathrm{SrTiO}_{3}$ was attributed by Baer to the Zeeman splitting of the fundamental absorption edge resulting from the $\mathrm{p}$-d charge transfer transition. He associated the transition energy $(3.4 \mathrm{eV})$ deduced from the theoretical fitting with the shoulder observed at $3.4 \mathrm{eV}$ in the reflectivity spectrum reported by Cardona. ${ }^{8)}$

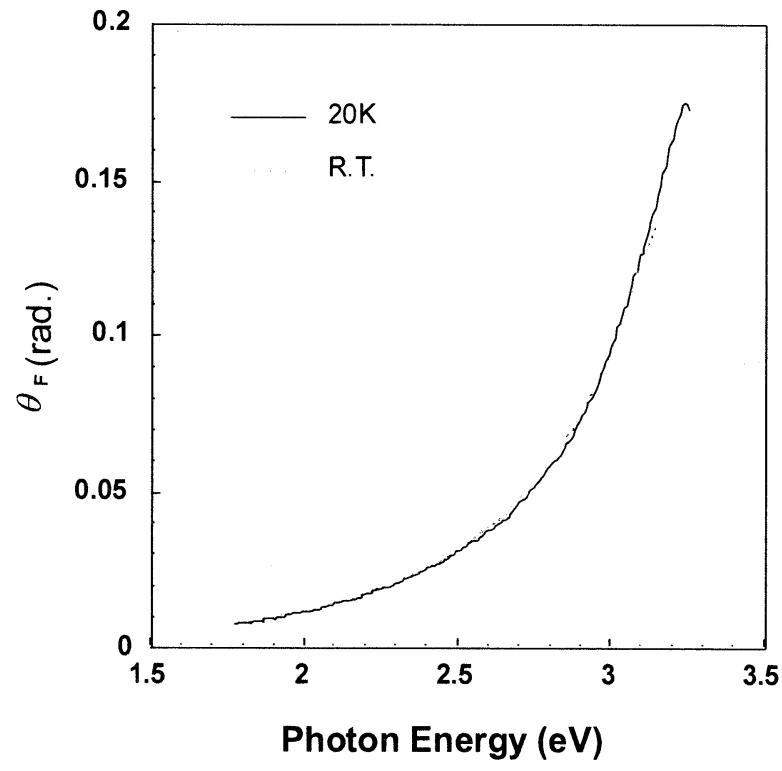

Fig. 3 Faraday rotation spectra of the $\mathrm{SrTiO}_{3}$ crystal measured at $20 \mathrm{~K}$ (solid curve) and room temperature (dotted curve).

We measured a reflectivity spectrum at room temperature between 0.5 and $30 \mathrm{eV}$ in the same sample for which Faraday effect was evaluated. Reflectivity spectrum is shown in Fig. 4. From the reflectivity spectra, real and imaginary parts of the dielectric functions were calculated using Kramers-Kronig relation. To determine appropriate extrapolation parameters for this analysis we used spectra of refractive constant $n$ and extinction constant $\kappa$ determined by spectroscopic ellipsometry. The resulting dielectric functions are given in Fig. 5. Here we only show spectra for energies below $10 \mathrm{eV}$ since the Faraday effect of this crystal is essentially related to the fundamental absorption region.

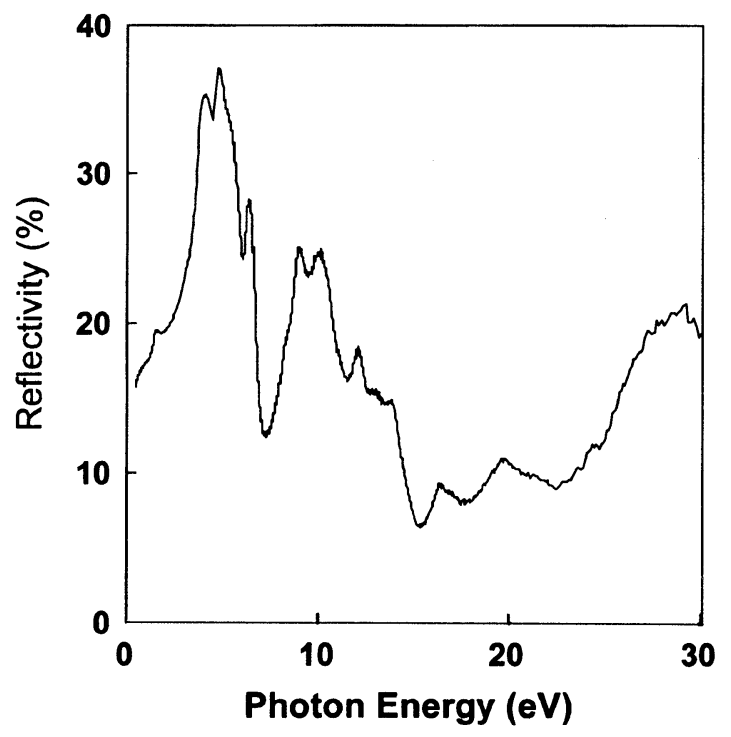

Fig. 4 Reflectivity spectrum of a $\mathrm{SrTiO} 3$ single crystal measured using synchrotron radiation. 


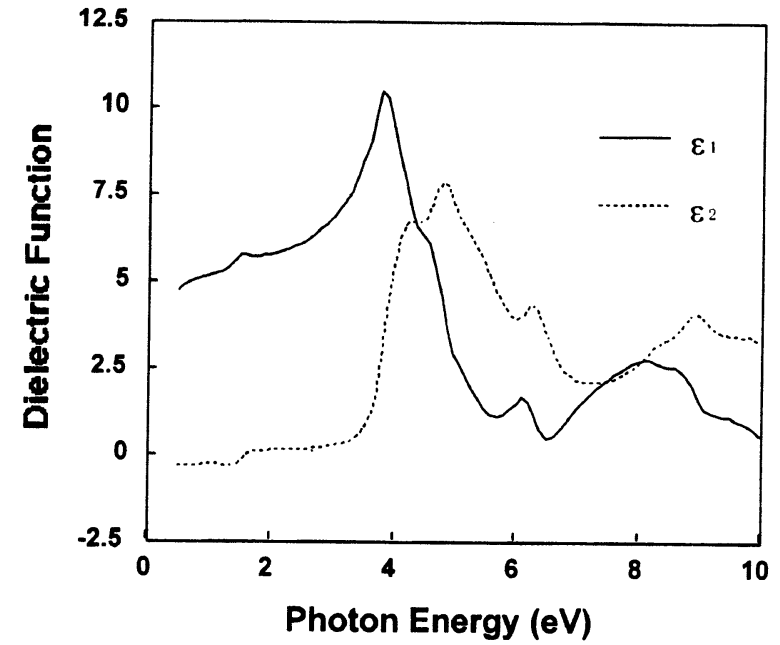

Fig. 5 Real and imaginary part of dielectric functions of $\mathrm{SrTiO}_{3}$ single crystal.

Contrary to Cardona's data we did not observe any structures around $3.4 \mathrm{eV}$ in the spectrum of the imaginary part of the dielectric function corresponding to the absorption spectrum. The first absorption peak in our spectrum is found at $4.2 \mathrm{eV}$, which shows a good correspondence with the energy position $(4.19 \mathrm{eV})$ of the center of dispersion determined by the analysis of the Faraday rotation spectrum. We suspect that the $3.4 \mathrm{eV}$ structure observed by Cardona is not an intrinsic one but due to defects or impurities.

\section{Conclusion}

Magnetooptical spectra were measured in single crystals of $\mathrm{SrTiO}_{3}$. Comparatively large temperature-independent Faraday rotation was obtained.
Figure of merit of $F=3.85 \mathrm{deg} / \mathrm{dB}$ is obtained at $400 \mathrm{~nm}$ for applied field of $1 \mathrm{~T}$. Further reduction of growth-induced residual absorption and improvement of surface treatment is required to get device quality materials. Fabrication of a short-wavelength isolator using $\mathrm{SrTiO}_{3}$ is under investigation. Details will be reported in later publications.

The center of dispersion for Faraday rotation was determined as $4.19 \mathrm{eV}$ from the analysis of the spectrum. The energy is consistent with the lowest energy peak position of $4.2 \mathrm{eV}$ in the imaginary part of the dielectric function obtained from the reflectivity spectrum

\section{Acknowledgements}

Authors are very grateful to Dr. M. Fujisawa of Synchrotron Laboratory, Institute of Solid State Physics, University of Tokyo for his help in VUV reflectivity measurements. They also thank Prof. T. Saito of Tokyo University of Agriculture and Technology for measurement of spectroscopic ellipsometry.

\section{References}

1) T. Okuda: J. Appl. Phys. 67, 4944 (1990).

2) K. Onodera. T. Matsumoto and M. Kimura: Electron Lett. 30, 1954 (1994)

3) R. Wolfe. A. J. Kurtzig and R. C. LeCraw: J. Appl. Phys. 41, I218(1970).

4) W.S.Baer: J. Phys. Chem. Solids, 28, 677 (1967).

5) A. Feldman. W. S. Brower and D. Horowitz: Appl. Phys. Lett. 16, 201 (1970)

6) K. Sato: Jpn. J. Appl. Phys. 20, 2403 (1981).

7) A.M. Clogston: J. Appl. Phys. 31, Suppl. 198 (1960).

8) M.Cardona: Phys. Rev. 140, A651 (1965).

Received March 31, 2002; Accepted July 12, 2002. 